\title{
Geschlechtsidentitäten im Wandel
}

\section{Gender identities in transition}

\author{
Esther Strittmatter ${ }^{1}$ und Martin Holtmann² \\ Gesundheitszentrum Walstedde, Tagesklinik GmbH, Drensteinfurt \\ LWL-Universitätsklinik Hamm der Ruhr-Universität Bochum, Hamm
}

\begin{abstract}
Zusammenfassung: Die Anzahl der Kinder und Jugendlichen, die sich aufgrund von Geschlechtsinkongruenzerleben und/oder Geschlechtsdysphorie in den Kliniken für Kinder- und Jugendpsychiatrie und Psychotherapie bzw. den vorhandenen Trans-Spezialsprechstunden vorstellen, ist in den letzten Jahren deutlich gestiegen. Sowohl national als auch international findet ein Diskurs über die psychotherapeutischen und medizinischen Gesundheitsbedarfe dieser jungen Menschen sowie die zugrunde liegende ethische Auslegung der Problematik statt. Die divergierenden Expertenmeinungen und fachlichen Verunsicherungen spiegeln sich nicht zuletzt in der unzureichenden psychotherapeutischen und psychiatrischen Versorgung für die geschlechtlich nonkonformen und geschlechtsdysphorischen Kinder und Jugendlichen wider. Der vorliegende Artikel gibt einen Überblick über die verfügbaren epidemiologischen Daten, den gesellschaftlichen, rechtlichen und medizinischen Paradigmenwechsel, die Entwicklungen in den neuen diagnostischen Klassifikationssysteme (DSM-5, ICD-11) sowie über wichtige Aspekte der AWMF-S3-Leitlinie „Geschlechtsinkongruenz, Geschlechtsdysphorie und Trans-Gesundheit“ für das Erwachsenenalter. Auf dieser Basis werden der aktuelle Diskurs und die ethischen Dilemmata im Kindes- und Jugendalter dargestellt sowie die sich daraus ergebenden Herausforderungen für die Kinder- und Jugend psychiatrie und Psychotherapie skizziert.
\end{abstract}

Schlüsselwörter: Trans, Geschlechtsinkongruenz, Geschlechtsdysphorie, Kinder und Jugendliche, ethische Dilemmata, therapeutische Herausforderungen

\section{Gender identities in transition}

Abstract: In recent years, the healthcare system has been confronted with an increasing number of children and adolescents with gender nonconformity, gender incongruence, and gender dysphoria. Medical professionals are still debating how to interpret this phenomenon and how best to meet the healthcare needs of this diverse group of young people. Meanwhile, the transgender and gender nonconforming youths themselves face enormous challenges in finding appropriate support and treatment in the mental healthcare system. This article reviews the available epidemiological data, the paradigm shift in the social, legal, and medical systems, the developments in diagnostic classifications (DSM-5, ICD-11) as well as important aspects of the AWMF S3 guideline for adults with gender incongruence and gender dysphoria. In addition, it describes the complexity of working with transgender, gender nonconforming, and gender-questioning youth in the context of the current discourse and the underlying ethical dilemmas. In conclusion, this article outlines the challenges facing child and adolescent psychiatry and psychotherapy in this complex environment.

Keywords: transgender, gender incongruence, gender dysphoria, children and adolescents, ethical dilemma, therapeutic challenge

Gegenwärtig findet sowohl in den nationalen und internationalen Fachzeitschriften als auch in der Populärwissenschaft und der Öffentlichkeit ein Diskurs über die deutlich steigende Anzahl von Kindern und Jugendlichen statt, die ihre empfundene Geschlechtsidentität im Widerspruch zu der ihnen zugeschriebenen Geschlechtszugehörigkeit erleben (u.a. Arcelus et al., 2015; De Graaf, Giovanardi, Zitz \& Carmichael, 2018; Hummel, 2019; Louis, 2020; Schwarzer, 2020; Spiewak, 2018). Die oftmals polarisierende Diskussion entzündet sich insbesondere an Jugendlichen, die in der Pubertät erstmals Geschlechtsinkongruenzerleben und Geschlechtsdysphorie beschreiben, sowie an der Frage einer pubertätsunter- drückenden und/oder geschlechtsangleichenden Hormonbehandlung im Jugendalter.

Da die psychotherapeutische und psychiatrische Versorgung für geschlechtsvariante, geschlechtlich nonkonforme und geschlechtsdysphorische Kinder, Jugendliche und ihre Familien unzureichend ist, haben betroffene $\mathrm{Fa}$ milien häufig einen langen Leidensweg und unzumutbare Wartezeiten hinter sich, bis sie an qualifizierte Beratungsund Behandlungsangebote gelangen.

Dabei spiegeln sich die Verunsicherungen und divergierenden Einschätzungen sowohl in den Familien als auch in der Kinder- und Jugendpsychiatrie und Psychotherapie wider. Die Fertigstellung des angemelde- 
ten S3-Leitlinienvorhabens „Geschlechtsdysphorie im Kindes- und Jugendalter, Diagnostik und Behandlung " wurde auf Ende 2020 verschoben. Unterdessen ist aus verschiedenen Quellen von Mitwirkenden der Leitliniengruppe zu lesen, dass „Fachdiskussionen [...] mit emotionaler Heftigkeit ausgetragen und [...] gegensätzliche Positionen und Annahmen" (Möller, Güldenring, Wiesemann \& Romer, 2018) aufweisen würden und dass „die Debatte komplett durchideologisiert“ (Louis, 2020) sei.

Es ist Zeit, zu einem unaufgeregteren Umgang mit dem Thema „Geschlecht“ zurückzukehren. Einerseits tragen die divergierenden Expertenmeinungen zu der großen Verunsicherung bei, andererseits weisen sie aber auch auf die Chance und Notwendigkeit hin, sich das aktuelle Fachwissen anzueignen und eine eigene ethische und therapeutische Haltung zu entwickeln.

\section{Was ist bekannt?}

Körperliches Geschlecht und geschlechtliche Identitäten sind endlos vielfältig und individuell. Geschlechtsidentität bezeichnet eine verinnerlichte, tief gefühlte Gewissheit über das eigene Geschlechtserleben, die nicht mit objektiven Kriterien gemessen werden kann (Güldenring, 2015). In Anlehnung an die S3-Leitlinie „Geschlechtsinkongruenz, Geschlechtsdysphorie und TransGesundheit“ wird der Begriff „Trans“ zur Anerkennung geschlechtlicher Vielfalt im Sinne eines inklusiven Oberbegriffs für Menschen gewählt, deren „Geschlecht nicht (bzw. nicht komplett und/oder dauerhaft) mit ihren körperlichen Merkmalen übereinstimmt" (Arbeitsgemeinschaft der Wissenschaftlichen Medizinischen Fachgesellschaften, 2019).

Im vorliegenden Artikel werden entsprechend der Richtlinien einer geschlechtergerechten Sprache des Hogrefe Verlages der Unterstrich benutzt, sofern Wortbestandteile der deklinierten Form enthalten sind, und Doppelnennungen verwendet, wenn dies nicht der Fall ist. So wird versucht, neben Männern und Frauen auch Menschen gerecht zu werden, die sich nicht (ausschließlich) als männlich oder weiblich erleben.

In der Vergangenheit haben Trans-Menschen durch die Medizin, die Rechtsprechung und die Gesellschaft viel Unrecht, Pathologisierung, Diskriminierung und Gewalt erfahren (Bundesministerium für Familie, Senioren, Frauen und Jugend, 2015; Collier, van Beusekom, Bos \& Sandfort, 2013; Grant et al., 2011; Valentine \& Shipherd, 2018). Das „Gesundheitssystem“ wird von ihnen als einer der am stärksten belastenden Bereiche erlebt (Güldenring, 2009; Hamm \& Sauer, 2014).

\section{Gesellschaftlicher und rechtlicher Paradigmenwechsel}

In den letzten Jahren hat sich in der Gesellschaft, der Rechtsprechung, der Gesetzgebung und im psychologischmedizinischen Diskurs ein grundlegender Paradigmenwechsel vollzogen. Soziologische Studien trugen zum Perspektivwechsel bei, indem sie den Fokus auf die kulturspezifisch zugewiesenen Geschlechtsrollenerwartungen und die Auswirkungen der Heteronormativität legten (Kessler \& Mc Kenna, 1978; Treibel, 2000). 1996 wurde vom Europäischen Gerichtshof ein Urteil zum Schutz der Geschlechtsidentität erlassen. 2009 und 2011 hat das Bundesverfassungsgericht schrittweise die Voraussetzungen für die Personenstandsänderungen (Ehelosigkeit, Operations- und Sterilisationszwang mit Unfruchtbarkeit) nach dem Transsexuellengesetz für verfassungswidrig erklärt. Das Bundesministerium für Familie, Senioren, Frauen und Jugend hat unlängst eine interministerielle Arbeitsgruppe „Inter- und Transsexualität" ins Leben gerufen, um die Lebenssituation von Menschen mit Varianten der Geschlechtsentwicklung und von Trans-Menschen zu verbessern und um Diskriminierungen in der Gesellschaft und Benachteiligungen in Medizin und Recht abzubauen (Bundesministerium für Familie, Senioren, Frauen und Jugend, 2015). Im Dezember 2019 stimmte das Bundeskabinett einem Gesetzentwurf von Gesundheitsminister Spahn zu, welcher Konversionstherapien auch an heranwachsenden, transgeschlechtlichen Menschen verbieten und unter Strafe stellen soll (vgl. die Stellungnahme der Deutschen Gesellschaft für Kinder- und Jugendpsychiatrie, Psychosomatik und Psychotherapie [DGKJP] dazu in diesem Heft auf den Seiten 169-170).

\section{Medizinisch-psychologischer Paradigmenwechsel}

Eine Wende in der Gesundheitsversorgung für Trans-Menschen stellte die siebte Version der Versorgungsempfehlungen (Standards of Care [SOC 7] for the Health of Transsexual, Transgender, and Gender-Nonconforming People) der World Professional Association for Transgender Health (WPATH) dar, die Geschlechtsinkongruenz als Ausdruck von Vielfalt (nicht Pathologie) und als ein heterogenes Phänomen mit pluralen Verlaufsformen begreift (Coleman et al., 2012). Auch in den Neufassungen der diagnostischen Manuale wurden die Begriffe „Störungen der Geschlechtsidentität" und „Transsexualität“ ersetzt. In der fünften Auflage des Diagnostic and Statistical Manual of Mental Disorders (DSM-5; American Psychiatric Association, 2013) wurde der Begriff „Geschlechtsdysphorie“ eingeführt und damit unterstrichen, dass nicht die Geschlechts- 
identität, sondern das Leiden an der erlebten Geschlechtsinkongruenz die Krankheit darstellt. Außerdem erfolgte dabei erstmals eine Aufweitung für andere, z.B. nonbinäre Geschlechtsidentitäten. Die angekündigte Veröffentlichung der ICD-11 (International Statistical Classification of Diseases and Related Health Problems) der Weltgesundheitsorganisation (WHO) führt die Entpathologisierung noch einen Schritt weiter, nimmt die neue Diagnose „Geschlechtsinkongruenz" aus dem F-Kapitel (Psychische und Verhaltensstörungen) und verortet sie unter „Zustände im Zusammenhang mit sexueller Gesundheit" (Drescher, Cohen-Kettenis \& Winter, 2012). Die Diagnosestellung setzt keinen gegenwärtigen Leidensdruck voraus, sondern kann auch bei antizipiertem Leidensdruck vergeben werden (Nieder, Briken \& Güldenring, 2016).

Ende 2018 wurde die evidenz- und konsensbasierte S3Leitlinie „Geschlechtsinkongruenz, Geschlechtsdysphorie und Trans-Gesundheit zur Diagnostik, Beratung und Behandlung " für das Erwachsenenalter verabschiedet (Arbeitsgemeinschaft der Wissenschaftlichen Medizinischen Fachgesellschaften, 2019). In dieser werden Alltagserfahrungen sowie eine psychotherapeutische Begleitung zwar empfohlen, stellen jedoch keine notwendige Voraussetzung für den Beginn körpermodifizierender Behandlungen dar. Ebenso gibt es keine festen zeitlichen Vorgaben für körpermodifizierende Behandlungen sowie fast keine absoluten Kontraindikationen (mit Ausnahme von Psychosen und dissoziativen Identitätsstörungen). Laut S3-Leitlinie stellt die hormonelle Behandlung von Behandlungssuchenden eine Therapiemethode dar, die „zur Reduktion von Geschlechtsinkongruenz und/oder Geschlechtsdysphorie und möglicher sekundär bedingter Symptome sowie zur Verbesserung der Lebensqualität beiträgt" (Evidenzgrad III, starker Konsens). Unter ärztlicher Supervision wird die hormonelle Behandlung bei Erwachsenen als kurz- und mittelfristig ,sichere Behandlungsmethode“ eingeschätzt (Evidenzgrad III, starker Konsens). Des Weiteren besteht ein „starker Konsens“, dass „modifizierende Behandlungen körperlicher Geschlechtsmerkmale" für Trans-Personen, die körpermodifizierende Behandlungen in Anspruch nehmen wollen, die „Therapie der ersten Wahl“ sind. Aus Respekt vor der autonomen, selbstverantwortlichen Trans-Person werden voll informierte, partizipative Entscheidungen angestrebt (Arbeitsgemeinschaft der Wissenschaftlichen Medizinischen Fachgesellschaften, 2019).

\section{Epidemiologie}

Angaben zur Prävalenz variieren je nach zugrunde gelegtem Definitionskriterium (Nieder et al., 2016; Köhler, Stern, Eyssel \& Nieder, 2019). Eine Metaanalyse kli- nischer Studien mit dem Behandlungsparadigma des Transsexualismus ergab eine Gesamtprävalenz von 4.6 / 100000 (2.6/100000 Frau-zu-Mann-Transitionen; 6.8/ 100000 Mann-zu Frau-Transitionen; Arcelus et al., 2015). Collin, Reisnner, Tangpricha und Goodman (2016) beschreiben eine Prävalenz von Trans-Selbstzuschreibungen von 355/100000, von transbezogenen Diagnosen von 6.8/100000 und von geschlechtsangleichenden Behandlungen (Hormone, Operationen) von 9.2/100 000. In einer niederländischen repräsentativen Querschnittsbefragung an 8064 Teilnehmenden einer nichtklinischen Stichprobe gaben $4.6 \%$ von den bei Geburt als Mann zugewiesenen Menschen an, sich ambivalent und $1.1 \%$ sich inkongruent gegenüber dem eigenen Geschlecht zu fühlen. Von den bei Geburt als Frau zugewiesenen Menschen gaben 3.2\% eine Geschlechtsambivalenz und $0.8 \%$ eine Geschlechtsinkongruenz an. Geschlechtsambivalenz oder -inkongruenz und der Wunsch nach geschlechtsangleichenden Maßnahmen traten bei $0.6 \%$ der bei Geburt als Mann und $0.2 \%$ der als Frau zugewiesenen Menschen auf. (Kuyper \& Wijsen, 2014). Auch andere Quellen weisen darauf hin, dass sich eine signifikante Anzahl von Trans-Menschen trotz Geschlechtsinkongruenzerleben partiell gegen geschlechtsangleichende Maßnahmen entscheidet (Beek, Kreukels, Cohen-Kettenis \& Steensma, 2015; Bockting, 2008; Eyssel, Koehler, Dekker, Sehner \& Nieder, 2017). In einer amerikanischen Onlinestudie mit 6456 Transgender oder geschlechtlich nonkonformen Studienteilnehmenden identifizierten sich $67 \%$ als binär (männlich oder weiblich) und $33 \%$ als ein anderes Geschlecht (z.B. nonbinär; Grant et al., 2011; ähnlich: EU-LGBT Survey; European Union Agency for Fundamental Rights, 2014). Es ist wahrscheinlich, dass auch nonbinäre Menschen verstärkt Hilfe in den Spezialsprechstunden suchen (Richards et al., 2016). Mehrere Studien weisen auf steigende Prävalenzzahlen (Arcelus et al., 2015; De Graaf et al., 2018) und eine Inversion des Geschlechterverhältnisses hin, mit mehr Jugendlichen, denen bei Geburt das weibliche Geschlecht zugewiesen wurde und die sich als trans-männlich bezeichnen (Aitken et al., 2015 Kaltiala-Heino, Sumia, Työläjärvi \& Lindberg, 2015; Zucker, 2017). Deutsche Studien mit entsprechend großen Fallzahlen liegen bislang nicht vor.

\section{Aktueller Diskurs und ethische Dilemmata im Kindes- und Jugendalter}

Laut einer Statistik des Londoner Gender Identity Development Service haben sich 2009/2010 insgesamt 97 Kinder und Jugendliche mit Geschlechtsdysphorie vorgestellt, 
im Jahr 2015/2016 waren es 1419 (Tavistock and Portman NHS Foundation Trust, 2016). Auch die deutschen Spezialambulanzen verzeichnen seit 2015 einen Anstieg der Inanspruchnahme, wobei die Neuvorstellungszahlen eher die Verfügbarkeit von Behandlungskapazitäten widerspiegeln als den Bedarf der Nachfrage. In den Spezialsprechstunden in Hamburg, Münster und München wurden die Wartelisten aufgrund unzumutbarer Wartezeiten zwischenzeitlich geschlossen.

\section{„Doing digital gender"?}

Im Jahr 2015 lag erstmals eine nahezu vollständige Ausstattung (99\%) der 12- bis 19-Jährigen mit Smartphones vor (JIM-Studie 2015; Medienpädagogischer Forschungsverbund Südwest, 2015). Einerseits hilft das Internet Informationen $\mathrm{zu}$ verbreiten, die Kommunikation $\mathrm{zu}$ erleichtern, Selbsthilfestrukturen effizienter aufzubauen und zu einem veränderten Selbstverständnis mit Positivbesetzung geschlechtlicher Vielfalt beizutragen. Andererseits steigen der Druck der gesellschaftlichen Schönheitsideale und die Aufforderungen zur Selbstoptimierung. Eine Untersuchung über weibliche Selbstinszenierung in den neuen Medien kam zu dem Ergebnis, dass Geschlechterdarstellungen in den erfolgreichsten YouTube-Kanälen und auf Instagram auf veraltet anmutenden Rollenstereotypen („Kinder, Küche, Kosmetik“) basieren (MaLisa Stiftung, 2019).

Bislang ist wenig bekannt, welche Auswirkungen die z.T. idealisierte Darstellung von Influencern in den sozialen Medien, die Erwartung der ununterbrochenen alltäglichen Selbstzurschaustellung sowie die Rückmeldungen aus der virtuellen Peergruppe auf die Identitäts- und Persönlichkeitsentwicklung der Kinder und Jugendlichen haben und ob dadurch auch Illusionen einer natürlich gegebenen Geschlechtsidentität erzeugt werden können. Auffallend in den Spezialsprechstunden für geschlechtsvariante Kinder und Jugendliche ist, dass Beschreibungen der Trans-Entwicklung bei einigen der Jugendlichen, die in der Pubertät erstmals Geschlechtsinkongruenzerleben und Geschlechtsdysphorie beschreiben, eher stereotyp anmutenden schematischen Erzählungen als Prozessen des inneren Gewahrwerdens gleichen. Allen diesen Jugendlichen ist gemeinsam, dass sie sich mit YouTube-Videos oder Darstellungen auf Instagram „,identifizieren“ konnten. Alternativ könnten es auch Versuche sein, den antizipierten Erwartungen an die Begutachtungsanleitung geschlechtsangleichender Maßnahmen bei Transsexualität des Medizinischen Dienstes des Spitzenverbandes Bund der Krankenkassen e.V. (2009) zu entsprechen (Nieder \& Richter-Appelt, 2011).

\section{Entwicklungsverläufe}

Das evidenzbasierte Wissen über die Entwicklungsverläufe von jungen Menschen, die sich erstmals in der Pubertät aufgrund von Geschlechtsinkongruenzerleben und Geschlechtsdysphorie in den Spezialsprechstunden vorstellen, ist sehr beschränkt. Faktoren, die die Beständigkeit (Persistenz) bzw. Unbeständigkeit (Desistenz) der Geschlechtsdysphorie sicher vorhersagen können, sind nicht bekannt (Steensma, Biemond, de Boer \& CohenKettenis, 2011). Von der Kindheit bis in das Pubertätsalter bleibt das Geschlechtsinkongruenzerleben in der Mehrzahl der Fälle nicht bestehen. Die Persistenzraten variieren zwischen $12 \%$ und $55 \%$, wobei die Fallzahlen der einzelnen Studien allesamt niedrig sind (Drummond, Bradley, Peterson-Badali \& Zucker, 2008; Steensma et al., 2011; Steensma, Kreukels, de Vries \& Cohen-Kettenis, 2013; Wallien \& Cohen-Kettenis, 2008). Während nicht alle Kinder mit Geschlechtsvarianz im Erwachsenenalter als Trans-Menschen leben, hatten nicht alle TransErwachsenen von Kindheit an geschlechtsvariantes Erleben (Drescher, Cohen-Kettenis \& Reed, 2016). Güldenring (2009) beschreibt bei den phasenspezifischen Konfliktthemen transsexueller Entwicklungswege, dass die innere Wahrnehmung des transsexuellen Erlebens bei „einer größeren Anzahl“" erstmals während der Pubertät stattfindet und mit großen Irritationen, Einsamkeitserleben, Isolation sowie internalisierter Transphobie verbunden sein kann. Kliniker_innen und Forscher_innen, die sich jahrzehntelang in der Begleitung und Behandlung von Trans-Menschen engagiert haben, betonen, dass sich Trans-Menschen jeden Alters nicht „wünschen“, dem anderen Geschlecht anzugehören. Sie haben sich ihr geschlechtliches Sosein nicht ausgesucht. Vielmehr handelt es sich um ein „tiefes Bedürfnis, eine lebenserhaltende Notwendigkeit“, entsprechend dem inneren Geschlechtsidentitätsempfinden leben zu können und anerkannt zu werden (Preuss, 2019b; Rauchfleisch, 2017). TransJugendliche können durch die Entwicklung des nichtstimmigen Geschlechtskörpers in der Pubertät in ihrer psychosexuellen Entwicklung massiv beeinträchtigt werden (Preuss, 2019a). Reaktive Folgeerkrankungen (z.B. Depressionen, Angsterkrankungen) werden u.a. mit dem Minoritätenstressmodell erklärt (Bockting, Miner, Swinburne Romine, Hamilton \& Coleman, 2013; Güldenring, 2009). Aus vielfältigen Studien ist bekannt, dass Trans-Kinder und -Jugendliche in fremdzugewiesenen Geschlechtsrollen ein deutlich höheres Risiko für emotionale Störungen, Verhaltensprobleme und psychische Auffälligkeiten haben als Trans-Kinder und -Jugendliche, die geschlechtlich selbstbestimmt und authentisch leben (Durwood, McLaughlin \& Olson, 2017; Möller et al., 2018; Roberts, Rosario, Corliss, Koenen \& Austin, 2012). 


\section{Begleiterkrankungen und Differenzialdiagnostik}

Die differenzialdiagnostische Abgrenzung und Einordnung von Begleiterkrankungen (siehe Arbeitsgemeinschaft der Wissenschaftlichen Medizinischen Fachgesellschaften, 2019, S. 26-36) ist bei Betroffenen, die erstmals im Jugendalter ihr geschlechtsdysphorisches Erleben thematisieren, schwierig und im Querschnitt nicht immer trennscharf möglich. Die Geschlechtsidentitätsproblematik kann Ausdruck einer pubertären Reifungskrise sein (Waddell, 1998). Sie kann passager im Rahmen allgemeiner Identitätsverunsicherung, begleitender Psychopathologie oder dem Wunsch nach Zugehörigkeit entstehen (Churcher Clarke \& Spiliadis, 2019). Besonders schwierig ist diese Einschätzung bei Jugendlichen, die zusätzlich zum Trans-Erleben ein niedriges Strukturniveau sowie begleitende psychiatrische Erkrankungen aufweisen (Arbeitsgemeinschaft der Wissenschaftlichen Medizinischen Fachgesellschaften, 2019; Bock et al., 2019; vgl. Seiffge-Krenke \& Schmeck, 2019; Seikowski, Gollek, Harth \& Reinhardt, 2008). Neben einer Übergangsidentität ist die Herausbildung einer nonbinären Geschlechtsidentität oder binären Trans-Entwicklung als Verlauf möglich (Preuss, 2019b).

In der Populärwissenschaft kursiert momentan der von Littmann nach Befragung von Eltern (2018/2019) geprägte Begriff der „Rapid-Onset Gender Dysphoria“ (ROGD). Die Vereinfachung, alle Jugendlichen, die erstmals in der Pubertät ihre Geschlechtsinkongruenz und/oder Geschlechtsdysphorie nach außen öffnen, einer „ROGD“-Gruppe zuzuordnen, wird der Komplexität der Thematik nicht gerecht und birgt die Gefahr, dass diese besonders vulnerable Betroffenengruppe stigmatisiert und nicht ernst genommen wird.

Zusammenfassend ist festzuhalten, dass bislang keine evidenzbasierten Kriterien existieren, die mit ausreichender Sicherheit die weitere Entwicklung der individuellen Geschlechtsidentität zuverlässig und trennscharf voraussagen könnten. Es werden dringend prospektive Forschungsansätze benötigt mit dem Ziel, unterschiedliche Subgruppen und Bedarfe zu differenzieren und bessere klinische Vorhersagen treffen zu können.

\section{Hormonbehandlung}

Weder national noch international besteht ein Konsens über den Beginn einer (pubertätsunterdrückenden und/ oder geschlechtsangleichenden) Hormonbehandlung sowie über die zugrunde liegende ethische Auslegung der Problematik (Churcher Clarke \& Spiliadis, 2019; Möller et al., 2018; Vrouenraets, Fredriks, Hannema, Cohen-Kettenis \& de Vries, 2015). Die Diskussion bewegt sich im Spannungsfeld zwischen dem Persönlichkeitsrecht auf geschlechtliche
Selbstbestimmung der Betroffenen auf der einen Seite und der Angst vor Fehlentscheidungen der Behandelnden auf der anderen Seite (Strauss \& Nieder, 2014). Bislang sind nur wenige prospektive Untersuchungen verfügbar. Studien mit großen Fallzahlen fehlen ebenso wie randomisiert kontrollierte Studien. Die Hormonbehandlung stellt einen Off-Label-Gebrauch dar. Neben der fehlenden sicheren Vorhersagbarkeit erscheint es problematisch, dass unter Expertinnen und Experten keine einheitlichen Entscheidungskriterien vorhanden $\mathrm{zu}$ sein scheinen. Vielmehr differiert die Entscheidung, welche Behandlungsangebote geschlechtsdysphorische Jugendliche erhalten je nach aufgesuchter Spezialsprechstunde, nach subjektiver Einschätzung der Untersuchenden vom „Passing“ in der angestrebten Geschlechtsrolle und nach der Persönlichkeitsstruktur der Betroffenen, d.h., wie glaubwürdig und konsistent das innere Erleben geschildert werden kann.

Korte, Beier und Bosinski (2016) warnen, dass es keine gesicherten Erkenntnisse gibt, wie sich eine Hormonbehandlung vor Abschluss der Pubertät auf die psychosexuelle Entwicklung und auf die Gehirnentwicklung auswirke und weisen auf die Gefahr hin, dass altersgerechte soziosexuelle Erfahrungen verhindert werden und TransEntwicklungen zementiert werden könnten. Auch muss berücksichtigt werden, dass sich gesundheitliche Probleme erst durch geschlechtsangleichende Maßnahmen und deren Komplikationen ergeben können. Befürworter_innen einer differenziellen Indikationsstellung von pubertätsunterdrückenden und geschlechtsangleichenden Hormonen verweisen auf die Gefahr von reaktiven psychischen Störungen beim Leben in der fremdzugewiesenen Geschlechtsrolle (Durwood et al., 2017; Möller et al., 2018; Roberts et al., 2012) und auf die im Langzeitverlauf überwiegend gute bis sehr gute gesundheitsbezogene Lebensqualität (Cohen-Kettenis, Delmarre-van der Waal \& Gooren, 2008). Sauer, Güldenring und Tuider (2015) unterstreichen, dass das Vorenthalten einer Behandlung zu weitreichenden lebenslangen Folgen mit erschwertem Passing, Mobbing und Stigmatisierungserfahrungen führen könne.

\section{Wie kann die Kinder- und Jugendpsychiatrie und Psychotherapie den Herausforderungen gerecht werden?}

Entwicklungen brauchen Zeit (Preuss, 2019b). Manchmal überholen die Veränderungen der Kinder und Jugendlichen die Anpassungsgeschwindigkeit der Eltern und des professionellen Hilfesystems. Jugendliche stellen sich 
häufig erst mit einem erheblichen Leidensdruck in den Spezialsprechstunden vor, nachdem sie bereits in allen Lebensbereichen den sozialen Rollenwechsel vollzogen und Fakten geschaffen haben. Junge Trans-Menschen sind in der Mitte unserer Gesellschaft angekommen. Die Kinderund Jugendpsychiatrie und Psychotherapie ist gefordert, sich mit den sich wandelnden gesellschaftlichen Realitäten auseinanderzusetzen und für Zustände, die mit einem krankheitswertigen Leidensdruck assoziiert sind, wohnortnahe, qualifizierte Beratungs- und Behandlungsangebote zu schaffen. Fachliche Voraussetzungen, die Gender-Spezialisten erfüllen sollten, sind in den SOC 7 (Coleman et al., 2012) und von der American Psychological Association (2015) formuliert worden.

Unabhängig von einem geschlechtsangleichenden Behandlungswunsch Betroffener und der Frage nach einer vorhandenen Indikation sollten Kinder und Jugendliche in ihren geschlechtlichen Selbstbeschreibungen gehört, geachtet und ernst genommen werden sowie in ihrem individuellen Ausdrucksbedürfnis gesehen werden (Möller et al., 2018). Auf der Basis einer vertrauensvollen therapeutischen Beziehung ist es möglich, auch Ängste, Zweifel und Ambivalenzen zu besprechen, um die Betroffenen in einer gesunden Persönlichkeits- und Identitätsentwicklung zu unterstützen. Insbesondere in der Pubertät bedarf es ausreichender Zeit für die Auseinandersetzung mit dem Unwohlsein im eigenen Körper, für die Exploration der Geschlechtsidentität und die Überprüfung der Lebbarkeit der individuellen Geschlechtsrollenvorstellungen. Eine realistische Einschätzung der Möglichkeiten und Grenzen geschlechtsangleichender Behandlungen sowie der mit ihnen verbundenen gesundheitlichen Risiken ist unabdingbar. Dies beinhaltet auch eine Auseinandersetzung mit dem eigenen Körper, der eigenen Sexualität und Fertilität.

Psychotherapie eröffnet die Möglichkeit, in einem geschützten Rahmen geschlechtliches Empfinden als existenzielles Bedürfnis wertzuschätzen und sich mit der individuellen geschlechtlichen Identität auseinanderzusetzen (Möller et al., 2018; Preuss, 2019a). Kinder und Jugendliche sollten ergebnisoffen und Ich-stärkend begleitet werden. Dabei sollten anstehende Entwicklungsaufgaben, ggf. begleitende Psychopathologie, Persönlichkeitsstruktur und soziale Integration beachtet werden (Di Ceglie, 2009). Im Kindes- und Jugendalter ist eine Prozessdiagnostik (differenzialdiagnostische Verlaufsbeobachtung) erforderlich, um prognostische Einschätzungen mit größtmöglicher Sicherheit treffen zu können. Eltern brauchen Unterstützung, Unsicherheiten auszuhalten, ihr Kind unabhängig vom Geschlecht als liebenswerten Menschen wohlwollend zu akzeptieren und mit einer Wachstum ermöglichenden Haltung zu begleiten, was auch die gemeinsame kritische Auseinandersetzung mit unterschiedlichen Wahrnehmungen und Einschätzungen miteinschließt.
Da Geschlechtsidentität ein tiefes inneres, individuelles Erleben ist, das nicht objektiviert werden kann (Güldenring, 2015), erscheint die in der Populärwissenschaft aufgeworfene Frage nach „echten“ und „Neo-Transsexuellen" nicht hilfreich. Wichtiger ist es, den vorhandenen Leidensdruck und die psychosexuelle Entwicklung gemeinsam mit den Betroffenen und der zugehörigen Familie zu verstehen, die Selbstwahrnehmung und Fremdwahrnehmung abzugleichen, um zu einer realistischen Selbsteinschätzung zu gelangen. Psychotherapie kann helfen, die häufig initial undifferenzierten, geschlechtsdysphorischen Gefühlszustände, die beispielsweise aus Angst, Ekel, Scham, Wut und Neid bestehen können, besser zu verstehen und einzuordnen (Preuss, 2019a). Ziel ist es, die geschlechtsdysphorischen jungen Menschen in der psychosexuellen und Identitätsentwicklung so zu unterstützen, dass sie Kohärenz und Kontinuität im Kontext der sozialen Beziehungen entwickeln können.

Die Frage der Indikationsstellung einer (pubertätsunterdrückenden und/oder geschlechtsangleichenden) Hormonbehandlung muss sehr sorgfältig geprüft werden. Es handelt sich um eine Dilemmasituation, in der eine neutrale Position nicht möglich ist (Cohen-Kettenis et al., 2008), da auch eine Nichtentscheidung eine Entscheidung darstellt. Kurz- und langfristige Chancen und Risiken einer Behandlung müssen gegenüber einer Nichtbehandlung abgewogen werden. Entscheidungen müssen prospektiv getroffen werden, sie werden sich jedoch erst retrospektiv nach einer längeren Zeit als richtig oder falsch erweisen.

Basierend auf den Diskriminierungs- und Pathologisierungserfahrungen sowie dem oftmals langen Leidensweg von erwachsenen Trans-Menschen (Bundesministerium für Familie, Senioren, Frauen und Jugend, 2015; Sauer et al., 2015), den retrospektiven Berichten von Trans-Erwachsenen über die sichere Wahrnehmung der Geschlechtsdysphorie ab dem Kindes- und/oder Jugendalter (Güldenring, 2009), wurden Möglichkeiten einer frühzeitigen Hormonbehandlung sorgfältig geprüft und eingeführt (De Vries et al., 2014). In Anlehnung an das Amsterdamer Modell haben sich auch Spezialsprechstunden/ Ambulanzen an Universitätskliniken in Deutschland entwickelt. Allerdings hat der Wunsch, zur Prävention dieses Leidensweges beizutragen, sekundäre Folgeerkrankungen zu vermindern und die Lebensqualität zu verbessern in Kombination mit der deutlich steigenden und sich qualitativ verändernden Inanspruchnahmepopulation der Spezialambulanzen - zu einer zu niedrigschwelligen und unkritischen Indikationsstellung für (pubertätsunterdrückende und/oder geschlechtsangleichende) Hormonbehandlungen geführt. Nun geht es darum, im Jugendalter aus dem Verfügbarkeitsmodus in einen kritischen Resonanzmodus zurückzukehren (Rosa, 2019), indem sorgfältig gemeinsam geprüft wird, ob es sich um eine Form der 
lebenslang persistierenden, tiefgreifenden, nichtveränderbaren, quälenden Geschlechtsdysphorie handelt, bei der es ohne geschlechtsangleichende Maßnahmen keine Linderung gibt. Das Grundprinzip, dass „eine geschlechtsangleichende Hormonbehandlung nur dann indiziert werden sollte, wenn alle Beteiligten (Patientinnen und Patienten, beide Eltern, alle behandelnden Ärztinnen und Ärzte und Psychotherapeutinnen und -therapeuten inkl. Kinder- und Jugendpsychiaterinnen und -psychiater, Gender Spezialistinnen und Spezialisten, pädiatrische Endokrinologinnen und Endokrinologen) darin übereinstimmen, dass eine Nichtbehandlung den Leidensdruck verschlimmern und zu einem absehbar größeren FolgeSchaden führen würde, als die Folge des Restrisikos einer sich möglicherweise später zeigenden Fehlindikation" (Preuss, 2019a), sollte angestrebt werden. Als Arbeitsund Verständnismodell für die Arbeit mit Trans-Jugendlichen und ihren Familien ist das (in der SOC 7 gestrichene) Prinzip der grundsätzlichen Eignung („elegibility“) und des richtigen Zeitpunktes („readiness") für geschlechtsangleichende Maßnahmen hilfreich, um den Möglichkeitsraum für die Entwicklung von Sicherheit und Einwilligungsfähigkeit zu vergrößern. Da Geschlechtsidentität einen der zentralen Aspekte von Würde und Selbstbestimmung darstellt (Yogyakarta Prinzipien, 2006) und die Einschätzung des prospektiven Nutzens und Schadens in hohem Maße von der Bewertung der Betroffenen abhängt, geht es nicht darum, für die Betroffenen Entscheidungen zu treffen. Vielmehr sollen die Jugendlichen und ihre Eltern mithilfe der therapeutischen Begleitung befähigt werden, auf der Basis des vorhandenen Wissens partizipative, informierte Entscheidungen treffen zu können (Möller et al., 2018). Therapeutinnen und Therapeuten wiederum dürfen in jedem Einzelfall nur Indikationen stellen, wenn sie diese auch ethisch vertreten können. Sie sind gefordert, ihre ggf. vorhandenen Bedenken transparent und wertschätzend mit den Betroffenen und deren Familien zu besprechen.

\section{Entwicklungsaufgaben}

Für den Aufbau einer wohnortnahen Versorgung bedarf es qualifizierter Fort- und Weiterbildungsangebote zu den Themen Geschlechtsidentitätsentwicklung und Transgeschlechtlichkeit. Seit 2019 findet in Hamburg ein erster akkreditierter Fortbildungsgang "Geschlechtsdysphorie, Geschlechtsinkongruenz, Trans- und Intergeschlechtlichkeit" nach den Richtlinien der Deutschen Gesellschaft für Sexualforschung statt. Durch diesen sind bereits neue qualifizierte Versorgungsangebote (z.B. am Universitätsklinikum Schleswig-Holstein, im Gesundheitszentrum Walstedde sowie in psychiatrischen und psychotherapeu- tischen Praxen für Kinder, Jugendliche und Erwachsene) entstanden. Das Thema Transgeschlechtlichkeit sollte zusätzlich stärker in die Curricula der Facharztweiterbildung und psychotherapeutischen Weiterbildungsinstitute mit aufgenommen werden.

Die aktuelle Befundlage zeigt, dass dringend prospektive Forschungsarbeiten benötigt werden, um Subgruppen besser voneinander abgrenzen zu können sowie mögliche Verläufe (Desistenz- und Persistenzraten) und das Ansprechen auf unterschiedliche Behandlungselemente besser vorhersagen zu können. Es sollte dargelegt werden, nach welchen Parametern welches Therapieangebot eröffnet wird und wie die Angemessenheit des Therapieangebotes überprüft werden kann. Neben dem Erfahrungswissen von erwachsenen Trans-Menschen, die retrospektiv ihre Entwicklung betrachten, wären auch Veröffentlichungen von nicht erfolgreichen Behandlungsverläufen sowie von der Gruppe von Jugendlichen hilfreich, die sich zu einer Re-Transition entscheiden. Um ausreichende Fallzahlen zu erzielen, wäre es ferner wünschenswert, konkurrenzbedingte Abgrenzungen zwischen den einzelnen Spezialzentren abzubauen sowie eine deutschlandweite Basiserhebung und vernetzte Forschung voranzubringen.

Neben dem Diskurs über die Veränderung von Geschlechtsidentitäten und über die Zunahme von Behandlungsanfragen wurde in den letzten Jahren zu wenig beachtet, welche inhaltlichen psychotherapeutischen Behandlungsbedarfe Trans-Kinder und -Jugendliche haben und in welchem Setting diese angeboten werden sollten. In der psychotherapeutischen Begleitung werden das direkte Spüren und Erleben des Körpers und die Sexualität häufig vernachlässigt. Da Trans-Sein durch ein dysphorisches Erleben der eignen Körperlichkeit gekennzeichnet ist, sind mehr transsensible, körpertherapeutische Angebote vonnöten (Hahne \& Stern, 2019).

Des Weiteren gelingt ein glückliches und erfülltes Leben von Kindern und Jugendlichen in der individuellen geschlechtlichen Identität insbesondere dann, wenn sie vorbehaltlos in tragenden familiären und erweiterten sozialen Beziehungen angenommen werden (Güldenring \& Sauer, 2017). Im Kindes- und Jugendalter sind daher systemische Angebote von besonderer Bedeutung, die vornehmlich die Familie, aber auch das erweiterte soziale Umfeld mit berücksichtigen. Erste erfolgversprechende, multifamilientherapeutische Ansätze sind dabei in den letzten Jahren etabliert worden und sollten weiter evaluiert werden (Strittmatter, 2017). Zusätzlich können Selbsthilfe, Peerberatung und Projekte wie beispielsweise „Trans - ja und?" helfen, das Gefühl von Selbstwert und Akzeptanz zu stärken und eine verbesserte Teilhabe an der Gesellschaft zu ermöglichen.

Die Arbeit mit transgeschlechtlichen Kindern, Jugendlichen und ihren Familien stellt hohe ethische und therapeu- 
tische Anforderungen an die Behandelnden (Möller et al., 2018). Gender-Spezialistinnen und -Spezialisten brauchen Vernetzung, die Möglichkeit zur Einholung von Zweitmeinungen aus anderen Zentren und Supervision. Es wäre daher wünschenswert, wenn die vorhandenen Spezialsprechstunden an den Universitätskliniken jenseits von Fortbildungsangeboten auch Kooperationsmöglichkeiten anbieten würden. Nicht zuletzt kann geschlechtsbezogene Selbsterfahrung Therapeutinnen und Therapeuten helfen, zu lernen, wie die möglicherweise im therapeutischen Beziehungsraum stehende Verwirrung zu verstehen ist.

Beim Thema Geschlecht greifen Natur-, Geistes- und Kulturwissenschaften in besonderer Art und Weise ineinander. Interdisziplinäre Forschungsansätze sind daher genauso wünschenswert wie die Klärung der Fragen: Wo beginnt die Rolle der Medizin? Und wo hört sie auf? In den letzten Jahren sind durch den gesellschaftlichen Diskurs, die Möglichkeiten des Internets, die Rechtsprechung, die Gesetzgebung und die Entpathologisierung im medizinisch-psychologischen Diskurs wichtige Schritte für die Achtung und Akzeptanz der geschlechtlichen Selbstbestimmung erreicht worden. Dabei sollten auch die den therapeutischen Kontroversen zugrunde liegenden ethischen Fragen diskutiert werden.

Am 19. Februar 2020 fand eine erste öffentliche Veranstaltung auf Einladung des Deutschen Ethikrates in der Reihe „Forum Bioethik“ zum Thema Trans-Identität bei Kindern und Jugendlichen statt. In der Folge wurden Ad-hoc-Empfehlungen zu ethischen Grundsätzen in der Begleitung und Behandlung von transidenten Kindern und Jugendlichen veröffentlicht (siehe Deutscher Ethikrat, 2020).

Gleichsam entsteht momentan der Eindruck, dass durch die Suche von Menschen nach einer passenden Selbstbeschreibung (u.a. agender, bigender, demigender, intergender, neutrosis, trigender, genderfuck, stargender, LSBATIQQP+; Queer Lexikon, 2020) und der damit verbundenen wechselseitigen $\mathrm{Ab}$ - und Ausgrenzung weniger statt mehr geschlechtliche Freiheit und Toleranz zu entstehen scheint. Die erhöhte Inanspruchnahme von Trans-Spezialambulanzen unterstreicht deutlich die Notwendigkeit der Auseinandersetzung mit der geschlechtlichen Identität, den kulturspezifischen Geschlechtsrollenerwartungen und den pubertären Veränderungen als wichtige Aufgabe auch für unser Fach.

\section{Literatur}

Aitken, M.A., Steensma, T.D., Blanchard, R., VanderLaan, D.P., Wood, H., Fuentes, A .et al. (2015). Evidence for an altered sex ratio in clinic-referred adolescents with gender dysphoria. Journal of Sexual Medicine, 12, 756-763.
American Psychiatric Association. (2013). Diagnostisches und Statistisches Manual Psychischer Störungen DSM-5: Deutsche Ausgabe (P. Falkei \& H.-U. Wittchen, Hrsg). Göttingen: Hogrefe.

American Psychological Association. (2015). Guidelines for psychological practice with transgender and gender non-conforming people. American Psychologist, 70, 832-864.

Arbeitsgemeinschaft der Wissenschaftlichen Medizinischen Fachgesellschaften e.V. (2019). Geschlechtsinkongruenz, Geschlechtsdysphorie und Trans-Gesundheit: S3-Leitlinie zur Diagnostik, Beratung und Behandlung. AWMF-Register-Nr. 138/ 001. Version 1.1, Stand: 22.02.2019.

Arcelus, J., Bouman, W.P., van den Norortgate, W., Claes, L., Witcomb, G. \& Fernandez-Aranda, F. (2015). Systematic review and meta-analysis of prevalance studies in transsexualism. European Psychiatry, 30, 807-815.

Beek, T.F., Kreukels, B.P., Cohen-Kettenis, P.T. \& Steensma, T.D. (2015). Partial treatment requests and underlying motives of applicants for gender affirming interventions. Journal of Sexual Medicine, 12, 2201-2205.

Bock, A., Huber, E., Müller, S., Henkel, M., Sevecke, K., Schopper, A. et al. (2019). Psychisches Strukturniveau im Jugendalter und der Zusammenhang mit späterer psychischer Erkrankung eine Langzeitstudie. Zeitschrift für Kinder- und Jugendpsychiatrie und Psychotherapie, 47, 400-410.

Bockting, W.O. (2008). Psychotherapy and the real-life experience: From gender dichotomy to gender diversity. Sexologies, 17, 211-224.

Bockting, W.O., Miner, M.H., Swinburne Romine, R.E., Hamilton, A. \& Coleman, E. (2013). Stigma, mental health, and resilience in an online sample of the US transgender population. American Journal of Public Health, 103, 943-951.

Bundesminsterium für Familie, Senioren, Frauen und Jugend. (2015). Gutachten: Begrifflichkeiten, Definitionen und disziplinäre Zugänge zu Trans- und Intergeschlechtlichkeiten. Begleitmaterial zur Interministeriellen Arbeitsgruppe Inter- \& Transsexualität, Bd. 1. Verfügbar unter https://www.bmfsfj.de/ blob/93956/ba3f7d5070103da9f2b62d08b23b2bac/imagband-1-gutachten-begrifflichkeiten-data.pdf

Churcher Clarke, A. \& Spiliadis, A. (2019). „Taking the lid off the box": The value of extended clinical assessment for adolescents presenting with gender identity difficulties. Clinical Child Psychology and Psychiatry 24, 338-352.

Cohen-Kettenis, P.T., Delmarre-van der Waal, H.A. \& Gooren, L.J.G. (2008). The treatment of adolescent transsexuals: Changing insights. Journal of Sexual Medicine, 5, 1892-1897.

Coleman, E., Bockting, W., Botzer, M., Cohen-Kettenis, P.T., DeCuypere, G., Feldman, J. et al. (2012). Standards of care, for health of transsexuals, transgender, and gender nonconforming people. International Journal of Transgenderism, 13, 165-232.

Collier, K.L., van Beusekom, G., Bos, H.M.W. \& Sandfort, T.G.M. (2013). Sexual orientation and gender identity/expression related peer victimization in adolescence: A systematic review of associated psychosocial and health outcomes. Journal of Sexual Research, 50, 299-317.

Collin, L., Reisnner, S. L., Tangpricha, V. \& Goodman, M. (2016). Prevalence of transgender depends on the „case“ definition: A systematic review. Journal of Sexual Medicine, 13, 613-626.

De Graaf, N. M., Giovanardi, G., Zitz, C. \& Carmichael, P. (2018). Sex ratio in children and adolescents referred to the Gender Identity Development Service in the UK (2009-2016). Archives of Sexual Behavior, 47, 1301-1304.

Deutscher Ethikrat. (2020). Trans-Identität bei Kindern und Jugendlichen. Therapeutische Kontroversen-ethische Orientierungen. Ad-hoc-Empfehlungen. Verfügbar unter https://www. ethikrat.org/fileadmin/Publikationen/Ad-hoc-Empfehlungen/ deutsch/ad-hoc-empfehlung-trans-identitaet.pdf. 
de Vries, A.L.C., McGuire, J.K., Steensma, T.D., Wagenaar, E.C.F., Doreleijers, T.A.H. \& Cohen-Kettenis, P.T. (2014). Young adult psychological outcome after puberty suppression and gender reassignment. Pediatrics 134, 696-704.

Di Ceglie, D. (2009). Engaging young people with atypical gender identity development in therapeutic work: A developmental approach. Journal of Child Psychotherapy, 35, 3-12.

Drescher, J., Cohen-Kettenis, P.T. \& Reed, G. M. (2016). Gender incongruence of childhood in the ICD-11: Controversies, proposal, and rational. Lancet Psychiatry, 3, 297-304.

Drescher, .J, Cohen-Kettenis, P. \& Winter, S. (2012). Minding the body: Situating gender identity diagnosis in the ICD-11. International Review of Psychiatry, 24, 568-577.

Drummond, K.D., Bradley, S.J., Peterson-Badali, M. \& Zucker, K.J. (2008). A follow-up study of girls with gender identity disorder. Developmental Psychology, 44, 34-45.

Durwood, L., McLaughlin, K.A. \& Olson, K. R. (2017). Mental health and self-worth in socially transitioned transgender youth. Journal of the American Academy of Child \& Adolescent Psychiatry, 56, 116-123.e2.

European Union Agency for Fundamental Rights. (2014). Being trans in the European Union. Comparative analysis of EU LGBT survey data. Verfügbar unter https://fra.europa.eu/en/publica tion/2014/being-trans-eu-comparative-analysis-eu-lgbt-sur vey-data

Eyssel, J., Koehler, A., Dekker, A., Sehner, S. \& Nieder, T.O. (2017). Needs and concerns of transgender individuals regarding interdisciplinary transgender healthcare: A non-clinical online survey. PLOS ONE, 12, e0183014.

Grant, J. M., Mottet, L.A., Tanis, J., Harrison, J., Herman, J. L. \& Keisling, M. (2011). Injustice at every turn: A report of the national transgender discrimination survey. Washington: National Center for Transgender Equality and National Gay and Lesbian Task Force.

Güldenring, A. (2009). Phasenspezifische Konfliktthemen eines transsexuellen Entwicklungsweges. Psychotherapie im Dialog, 10, 25-31.

Güldenring, A. (2015). A critical view of transgender health care in Germany: Psychopathologizing gender identity - Symptom of „disordered“ psychiatric/psychological diagnostics? International Review of Psychiatry, 27, 427-434.

Güldenring, A. \& Sauer, A. (2017). Trans*... inklusiv? Geschlechtsidentitäten in Medizin, Recht und Gesellschaft. In E. Diehl (Hrsg.), Teilhabe für alle?! Lebensrealitäten zwischen Diskriminierung und Partizipation (S. 231-257). Bonn: Bundeszentrale für politische Bildung.

Hahne, A. \& Stern, K. (2019). Trans*Körper*Wahrnehmung. Körperpraktische Methoden als Ergänzung zu Gesprächstherapie und Beratung. In M.N. Appenroth \& M. do Mar Castro Varela (Hrsg.), Trans \& Care. Trans Personen zwischen Selbstsorge, Fürsorge und Versorgung (S. 241-254). Bielefeld: Transcript.

Hamm, J. \& Sauer, A. (2014). Perspektivenwechsel: Vorschläge für eine menschenrechts- und bedürfnisorientierte Trans*-Gesundheitsversorgung. Zeitschrift für Sexualforschung, 27, 4-30.

Hummel, K. (2019). Einmal Mann sein, bitte. Frankfurter Allgemeine Sonntagszeitung. 8. Dezember 2019, Nr. 49, S. 11-12.

Kaltiala-Heino, R., Sumia, M., Työläjärvi, M. \& Lindberg, N. (2015). Two years of gender identity service for minors: Overrepresentation of natal girls with severe problems in adolescent development. Child and Adolescent Psychiatry and Mental Health, 9, 1-9.

Kessler, S.J. \& McKenna, W. (1978). Gender - an ethnomethological approach. New York: University of Chicago Press.

Köhler, A., Stern, K., Eyssel, J. \& Nieder, T.O. (2019). Zur Zweigeschlechtlichkeit und darüber hinaus. Identitäten, Körper und Behandlungsanliegen von trans Personen. Persönlichkeitsstörungen, 23, 101-113.
Korte, A., Beier, K.M. \& Bosinski, H.A.G. (2016). Behandlung von Geschlechtsidentitätsstörungen (Geschlechtsdysphorie) im Kindes- und Jugendalter - Ausgangsoffene psychotherapeutische Begleitung oder frühzeitige Festlegung und Weichenstellung durch Einleiten einer hormonellen Therapie? Sexuologie, 23, 117-132.

Kuyper, L. \& Wijsen, C. (2014). Gender identities and gender dysphoria in the Netherlands. Archives of Sexual Behavior, 43, 377-385.

Littman, L. (2018). Parent reports of adolescents and young adults perceived to show signs of a rapid onset of gender dysphoria. PLoS ONE, 13, e0202330 (Correction in: PLOS ONE [2019] 14, e0214157).

Louis, C. (2020). Emma Dossier (Sie Er Ich. Einfach das Geschlecht wechseln? Was richten wir da an? Interview mit A. Korte). Emma, Januar/Februar 2020, S. 50-61.

MaLisa Stiftung. (2019). Weibliche Selbst-Inszenierung in den neuen Medien. Geschlechterdarstellungen auf YouTube, Instagram und in Musikvideos. Verfügbar unter https://malisastif tung.org/geschlechterdarstellung-neue-medien/

Medienpädagogischer Forschungsverbund Südwest. (2015). JIMStudie 2015. Jugend, Information, (Multi-) Media. Basisuntersuchung zum Medienumgang 12- bis 19-Jähriger. Verfügbar unter https://www.mpfs.de/studien/jim-studie/2015/

Medizinischer Dienst des Spitzenverbandes Bund der Krankenkassen e.V. (2009). Grundlagen der Begutachtung - Begutachtungsanleitung - Geschlechtsangleichende Maßnahmen bei Transsexualität. Verfügbar unter https://www.mds-ev.de/filead min/dokumente/Publikationen/GKV/Begutachtungsgrundla gen_GKV/07_RL_Transsex_2009.pdf, abgerufen 26.01.2020.

Möller, B., Güldenring, A., Wiesemann, C. \& Romer, G. (2018). Geschlechtsdysphorie im Kindes- und Jugendalter. Kinderanalyse, 26, 228-263.

Nieder, T. O., Briken, P. \& Güldenring, A. (2016). Geschlechtsinkongruenz, -dysphorie und Trans*-Gesundheit, InFo Neurologie \& Psychiatrie, 18(12), 37-46.

Nieder, T.O. \& Richter-Appelt, H. (2011). Tertium non datur - either/ or reactions to transsexualism amongst health care professionals: The situation past and present, and its relevance to the future. Psychology and Sexuality, 2, 224-243.

Preuss, W.F. (2019a). Geschlechtsdysphorie, Transidentität und Transsexualität im Kindes- und Jugendalter. Diagnostik, Psychotherapie und Indikationsstellungen für die hormonelle Behandlung (2. akt. Aufl.). München: Ernst Reinhardt Verlag.

Preuss, W. F. (2019b). Trans*-Jugendliche brauchen Zeit, um Frauen und Männer zu werden. Drei Fallvignetten mit Anmerkungen zur Aufgabe der Psychotherapie bei transsexuellen Jugendlichen und Erwachsenen. Kinderanalyse, 27, 85-104.

Queer Lexikon. (2020). Glossar. Verfügbar unter https://queer-lexikon.net/category/queer-lexikon/glossar

Rauchfleisch, U. (2017). Transsexualität - Transidentität. Begutachtung, Begleitung, Therapie. Göttingen: Vandenhoeck \& Ruprecht.

Richards, C., Bouman, W.P., Seal, L., Barker, M.J., Nieder, T.O. \& T'Sjoen, G. (2016). Non-binary or genderqueer genders. International Review of Psychiatry, 28, 95-102.

Roberts, A. L., Rosario, M., Corliss, H. L., Koenen, K.C. \& Austin, S. B. (2012). Childhood gender nonconformity: A risk indicator for childhood abuse and posttraumatic stress in youth. Pediatrics, $129,410-417$

Rosa, H. (2019). Das Gespräch: Lob der Unverfügbarkeit. Philosophie Magazin, 4, 68-73.

Sauer, A., Güldenring, A. \& Tuider, E. (2015). Queering Trans*-Gesundheit: Auf dem Weg zu einer individualisierten, menschenrechtskonformen Gesundheitsversorgung. In P. Kolip \& K. Hurrelmann (Hrsg.), Handbuch Geschlecht und Gesundheit. Männer und Frauen im Vergleich (S. 420-432). Bern: Huber 
Schwarzer, A. (2020). Transsexualität 2020. Anpassung an die Rolle? Transsexualität 1984 Befreiung von der Rolle, Emma, Januar/Februar 2020, S. 6-7.

Seiffge-Krenke, I. \& Schmeck, K. (2019). Operationalisierte Psychodynamische Diagnostik im Kindes- und Jugendalter: Befunde zur Beziehungs- und Strukturachse. Zeitschrift für Kinderund Jugendpsychiatrie und Psychotherapie, 47, 385-387.

Seikowski, K., Gollek, S., Harth, W. \& Reinhardt, M. (2008). Borderline-Persönlichkeitsstörungen und Transsexualität. Psychiatrische Praxis, 35, 135-141.

Spiewak, M. (2018). Zwischen Kopf und Körper. Die Zeit, 48, S. 33-34.

Steensma, T.D., Biemond, R., de Boer, F. \& Cohen-Kettenis, P.T. (2011). Desisting and persisting gender dysphoria after childhood: A qualitative follow-up study. Clinical Child Psychology and Psychiatry, 16, 499-516.

Steensma, T.D., Kreukels, B.P.C., de Vries, A.L.C. \& Cohen-Kettenis, P.T. (2013). Gender identity development in adolescence. Hormones and Behavior, 64, 288-297.

Strauss, B.M. \& Nieder, T.O. (2014). Leitlinienentwicklung Geschlechtsdysphorie. Zeitschrift für Sexualforschung, 27, 1-3.

Strittmatter, E. (2017). Multifamiliengruppen für transidente Kinder, Jugendliche und ihre Familien. In E. Asen \& M. Scholz (Hrsg.), Handbuch der Multifamilientherapie (S. 123-133). Heidelberg: Carl-Auer.

Tavistock and Portman NHS Foundation Trust. (2016). Gender Identity Development Service Statistics. Verfügbar unter https://tavistockandportman.nhs.uk/documents/408/gids-servi ce-statistics.pdf

Treibel, A. (2000). Geschlecht als soziale Konstruktion: Ethnomethodologie und Feminismus. In A. Treibel (Hrsg.), Einführung in soziologische Theorien der Gegenwart (S. 133-153). Wiesbaden: VS Verlag für Sozialwissenschaften.

Valentine, S.E. \& Shipherd, J.C. (2018). A systematic review of social stress and mental health among transgender and gender non-conforming people in the United States. Clinical Psychology Review, 66, 24-38.

Vrouenraets, L.J., Fredriks, A. M., Hannema, S. E., Cohen-Kettenis, P.T. \& de Vries, M.C. (2015). Early medical treatment of children and adolescents with gender dysphoria: An empirical ethical study. Journal of Adolescent Health, 57, 367-373.

Waddell, M. (1998). Inside lives: Psychoanalysis and the growth of personality. London: England Kranac Books.

Wallien, M.S.C. \& Cohen-Kettenis, P.T. (2008). Psychosexual outcome of gender-dysphoric children. Journal of the American Academy of Child \& Adolescent Psychiatry, 47, 1413-1423.

Yogyakarta Principles. (2006). Principles on the application of international human rights law in relation to sexual orientation and gender identity. Verfügbar unter https://yogyakartaprinciples.org

Zucker, K.J. (2017). Epidemiology of gender dysphoria and transgender identity. Sexual Health, 14, 404-411.

\section{Interessenkonflikt}

Dr. Strittmatter leitet u.a. eine Trans-Ambulanz

im Gesundheitszentrum Walstedde.

\section{Dr. Esther Strittmatter}

Gesundheitszentrum Walstedde

Tagesklinik GmbH

Dorfstr. 9

48317 Drensteinfurt

Deutschland

esther.strittmatter@tagesklinik-walstedde.de

\section{Prof. Dr. Dr. Martin Holtmann}

LWL-Universitätsklinik Hamm der Ruhr-Universität Bochum Heithofer Allee 64

59071 Hamm

Deutschland

martin.holtmann@lwl.org

\section{AGNP-Psychopharmakologie-Tage} 19. - 20. Juni 2020, Mercure Hotel Moa Berlin

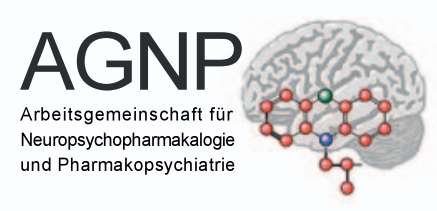

In diesem zweitägigen Intensivkurs wird sowohl Erwachsenen- als auch Kinder- und Jugendpschiatern sowie anderen interessierten Fachärzten und Psychologen von ausgewiesenen Experten ein umfassendes Wissen über alle Themen der Psychopharmakotherapie vermittelt. Der Kurs wird ohne Sponsoring der Pharmaindustrie veranstaltet.

Die Kosten inklusiver einer Bewirtungspauschale betragen $300 €(270 €$ für AGNP-Mitglieder).

Das Programm finden Sie auf der Homepage der AGNP. Die Anmeldung erfolgt online unter www.agnp.de 